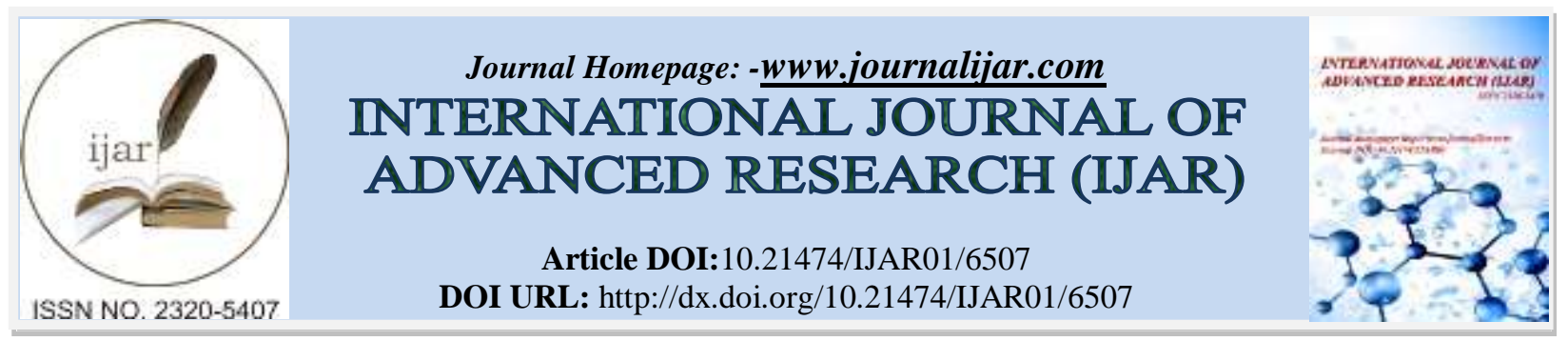

RESEARCH ARTICLE

\title{
QUANTITATIVE ETHNOBOTANICAL DOCUMENTATION OF THE MEDICINAL PLANTS USED BY THE INDIGENOUS MARING TRIBE OF CHANDEL DISTRICT OF MANIPUR, INDIA.
}

\author{
R. Nongmaithemand A. Kr. Das. \\ Laboratory of Ethnobotany \& Medicinal Plant Conservation, Department of Ecology \& Environmental Science, Assam \\ University, Silchar-788 011.
}

\section{Manuscript Info}

Abstract

Manuscript History

Received: 11 December 2017

Final Accepted: 13 January 2018

Published: February 2018

Copy Right, IJAR, 2018,. All rights reserved.

\section{Introduction:-}

Quantitative ethnobotanical study in Manipur:-

Ethnobotany and ethno-medicinal plant studies are recognized as the most viable method for identifying new medicinal plants and refocusing on those earlier reported for bioactive constituents (Abujam et al., 2012). Therefore, studies and documentation on ethnobotanical and traditional knowledge on medicinal plant uses has been considered as a high priority sometimes leading to the discovery of crude drugs (Cox and Ballick, 1994; Cox and Ballick, 1996; Dutta and Dutta, 2005; Hamil et al., 2000; Pieroni, 2000). Ethnobotanical knowledge of a particular community which includes all its socio-cultural activities of healthcare, food, timber, shelter, clothing as well as resource management \& conservation pattern was endemic and unique to that specific community only. The ethnic people are depended on the plants around him, made him to acquire knowledge of economic and medicinal properties of many plants by trial and error. Consequently, he became the storehouse of knowledge of many useful as well as harmful plants accumulated and enriched through generations and passed on from one generation to another without any written documents (Sur and Halder, 2002).

Quantitative methods in ethnobotany dated back to 1986 when Trotter and Logan for the first time used the informant consensus factor to the study of the relationship between the efficacy of the claimed herbal plants and their bio-activity. The concept of Use Value evaluating the significance of the particular plants was further developed by Phillips and Gentry (1993). Over the year interest in the application of quantitative methods to ethnobotanical data, testing different hypothesis to the relationship between plants and human has shown a steady growth and more researchers are focusing on incorporating varied quantitative methodology for data collection (Phillips et al., 1994; Reyes - Garcia et al., 2006)

Despite the advancement in ethnobotanical studies in recent years, the region lacks behind the documentation of such organized and quantitative ethnobotanical study. Therefore, an attempt has been made here and this can serve as baseline for future pharmacological studies.

Corresponding Author:-R. Nongmaithem. 


\section{Indigenous Maring tribe:-}

The Maring are among the oldest tribes of Manipur and settled in present Tengnoupal sub-division of Chandel district of Manipur, India. The name Maring was derived from two words 'Mi' (corrupted to Ma) meaning fire and 'Ring' meaning to start or produce. They are considered as one of the major tribes but taking the tribal as a whole this is one of the minor group considering their population. According to Census, 2011 report, the total population of Maring in the state stands as 26,424 while Chandel district alone record for 14,4182 Maring population. They are very skilled particularly in cane and bamboo work and the entire Manipur depend on them for beautiful yet useful items like Phiruk etc. Among the many distinctiveness and peculiarities of the tribe- "Blacken teeth" (Ha-sang in local term) was common amongst the elderly people and youth of the time. Knotted hairs or Murshoom on the front side of all the men-folk's forehead with several lines of hard red beads strings- Rulshum around their Murshoom is another peculiarity that set aside this tribe from the rest. They keep their hair long, gathered in a bunch somewhat like a horn rising from the front of the head. A tuft of frontal hair is tied into a knot. In the light of Christianity entering the community and modern education however, such practices are no longer encouraged and practiced. Gradually long hair of men-folk's for knotted hair or Murshoom are replaced today with plastic woven headgear. Therefore, such practices remain as oral traditional knowledge transferred from generation to generation.

The Maring are still predominantly shifting cultivators. The land on the steep hills called Pamlou was used for different types of cultivation. They are mainly forest dwellers and depend on available biodiversity in and around their settlement areas for food, medicine and comfort. Thus the ethnic Maring tribe was well acquainted with the role played by the plants and harbor a rich treasure on their utilization. This unwritten information was well transmitted and trapped through oral tradition from generation to generation often intermingling with their cultures, customs, traditions and taboos.

\section{Materials And Methods:-}

\section{Study site:-}

The state of Manipur is located in North Eastern part of India $\left(23^{\circ} 83^{\prime}-25^{\circ} 68^{\prime} \mathrm{N} / 93^{\circ} 03^{\prime}-94^{\circ} 78^{\prime} \mathrm{E}\right)$ and covers a total geographical area of $22,327 \mathrm{~km}^{2}$. An oval-shaped valley $(1,843 \mathrm{sq} . \mathrm{km})$ lies in the centre and is surrounded by series of mountains accounting to $90 \%$ of the total area. The state is divided into nine (9) administrative districts, viz Bishenpur, Imphal East, Imphal West, Thoubal in valley region and Churachandpur, Chandel, Senapati, Tamenglong, Ukhrul in hill region.

Chandel district with a total geographical area of $3,313 \mathrm{~km}^{2}$ lies in between $23^{\circ} 49^{\prime}-24^{\circ} 28^{\prime} \mathrm{N} / 94^{\circ} 09^{\prime}-94^{\circ} 31^{\prime} \mathrm{E}$ in the south- eastern part of the state of Manipur. This border district of the state neighbors Myanmar on the south, Ukhrul district on the east, Churachandpur district on the south and west, and Thoubal on the north. It is about $64 \mathrm{~km}$ from Imphal, the state capital. Formerly known as Tengnoupal district, the district is inhabited by several communities Anal, Lamkang, Kukis, Moyon, Monsang, Chothe, Thadou, Paite and Maring are the prominent tribes scattered all over the district. 


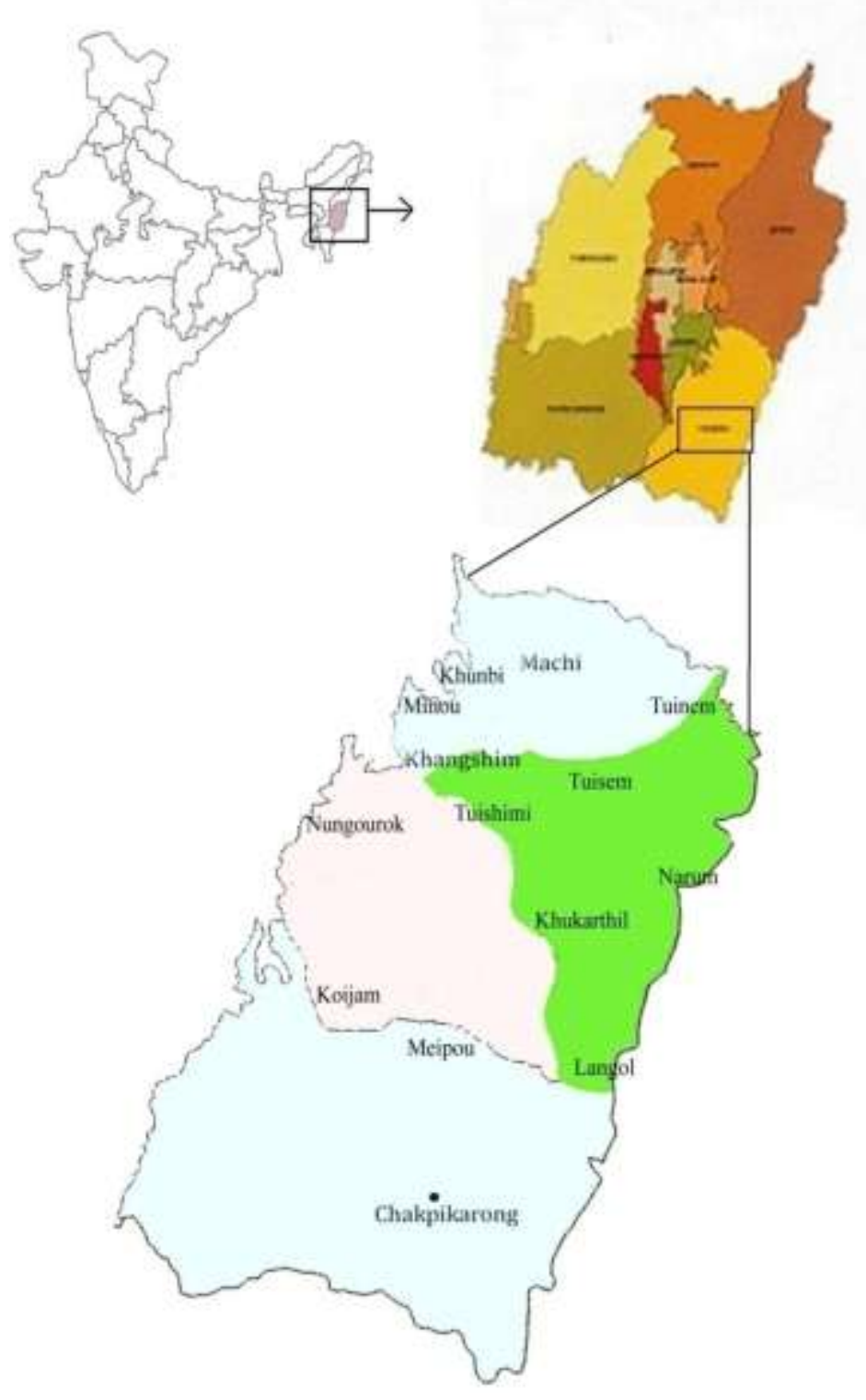

Fig. 1:-Maps showing the study site- Chandel District of Manipur

\section{Data Collection:-}

The present study was conducted during June 2012 to December 2016 in Manipur. Data collection and analysis was done through frequent survey programme based on semi-structured questionnaire. Prior informant consent (PIC) was obtained from local healers (Thim, Maibas), village chiefs, old folks, housewives etc. The age group of the informants falls between 30-80 years. Thirteen (13) sites were selected through random sampling. Informants totaling to 250 individual informants (176 males; 74 females) and twenty (20) key informants who have immense/ profound knowledge on plants were selected purposively for data analysis. This selection was aided by the village headman, Thim and other authorities.

\begin{tabular}{|c|c|c|}
\hline Parameter & Informant group & Number \\
\hline Gender & Male & 176 \\
& Female & 14 \\
\hline Age & Young ( $\leq 40$ years $)$ & 96 \\
\hline Educational status & Senior ( $\geq 40$ years $)$ & 68 \\
\hline
\end{tabular}




\begin{tabular}{|c|c|c|}
\hline & Illiterate & 182 \\
\hline Informants & Key informants & 20 \\
& General informants & 230 \\
\hline
\end{tabular}

Table 1:- Break-wise tabulation data of Informants (Maring group) of Chandel district, Manipur.

\section{Biological parameters, Species Description \& Classification:-}

Detailed morphological description of the documented plants was studied from mature vegetative and reproductive parts. All related data (synonyms, distribution, propagation and mode/ method of application, other uses) were also studied briefly. The scientific names along with their respective families, local name, parts used and mode of usage were recorded and highlighted. Identification was done based on available literatures (Hooker, 1875; Hooker, 1882; Deb, 1961; Li and Hedge, 1994; Singh et al., 2000). Classification, author citation and updated nomenclature are provided based on Brummit and Powell (1992), the Plant List (www. plantlist. org), the International Plant Names Index (http://www.ipni. org.), Bendiksby et al., (2011) and Angiosperm Phylogeny Group, APG III (2009). Specimens were collected, processed, identified and deposited in triplicates to Herbarium, Laboratory of Ethnobotany \& Medicinal Plant Conservation (AUS, Assam University, Silchar) and Manipur University Museum of Plants (MUMP).

\section{Data analysis:-}

Disease categories;-

The documented data of medicinal plants was grouped into 18 disease categories based on Cook (1995).

Table 2:-Ailments categories based on Cook (1995)

\begin{tabular}{|c|c|c|}
\hline Ailment Categories & Biomedical terms & Local terms \\
\hline Liver problems (LP) & Jaundice, hepatic complaint & Machu shang \\
\hline Circulatory System Disorders (CSD) & $\begin{array}{l}\text { Blood clotting, blood purification, } \\
\text { blood pressure }\end{array}$ & Hi dui \\
\hline Antidote (Ad) & Snake bite, Dog bite, bee sting & Thrul lei chik, Ui yei chik \\
\hline Endocrinal Disorder (ED) & Diabetes & Shim duilaknei \\
\hline Respiratory System Disorders (RSD) & $\begin{array}{l}\text { Asthma, bronchitis, cough, cold, } \\
\text { tuberculosis }\end{array}$ & Nilso tok, Bur chu \\
\hline Fever (Fr) & Fever & Shabang leh Reileh \\
\hline $\begin{array}{l}\text { Skeleton Muscular System Disorders } \\
\text { (SMSD) }\end{array}$ & $\begin{array}{l}\text { Bone fracture, body ache, } \\
\text { swellings, headache }\end{array}$ & Thru dikur, Luu kana \\
\hline Gastro intestinal disorders (GID) & $\begin{array}{l}\text { Dysentery, diarrhea, indigestion, } \\
\text { stomachache, constipation, ulcer, } \\
\text { intestinal worm }\end{array}$ & Uuk phe, uuk na \\
\hline Ear, Nose, Throat problem (ENT) & $\begin{array}{l}\text { Sinusitis, earache, epistaxis (Nose } \\
\text { bleeding), eye diseases, tonsillitis, } \\
\text { sore throat }\end{array}$ & Nahi da, dang na \\
\hline Dermatological infection (DI) & $\begin{array}{l}\text { Boils, scabies, rashes, } \\
\text { inflammation \& burns, ringworm }\end{array}$ & Yek, Mai thrai, Mei yei pok \\
\hline Kidney Stone (KS) & Kidney stone & Kaal rei lung lei \\
\hline Genito-urinary disorders (GUD) & $\begin{array}{l}\text { Urinary bladder stone, menstrual } \\
\text { disorder, leucorrhoea, Dysuria, } \\
\text { abortion, labor pain, pregnant } \\
\text { complicacies }\end{array}$ & Ae lakai dipur na \\
\hline Oral Care (OC) & $\begin{array}{l}\text { Toothache, gum complaints, mouth } \\
\text { ulcer }\end{array}$ & Ha chik nei \\
\hline Oncogenes $(\mathrm{Og})$ & Cancer, tumors & Cancer, Porbang \\
\hline Piles (P) & Piles & Dirang pum \\
\hline Deworming (Dw) & Worm Expulsion & - \\
\hline Smallpox (Sp) & Smallpox & - \\
\hline General Health $(\mathrm{GH})$ & Blood tonic & - \\
\hline
\end{tabular}




\section{Use Report:-}

When a plant was claimed to be effective in curing a particular ailment it was recorded as one use - report. A multiple use - reports can be recorded when more than one informants claimed the same plant for the same ailment.

\section{Use - Value (UV):-}

Use-Value or UV demonstrates the relative importance of species known locally (Phillips et al., 1994) which is based on the number of uses of a particular plant species and the number of informants that claimed the uses of the given plant. UV is calculated using the formula:

$\mathrm{UV}=\sum \mathrm{U} / \mathrm{N}$, where $\mathrm{UV}$ is the use value of a species, $\mathrm{U}$ is the number citations per species; $\mathrm{N}$ is the number of informants.

High UVs signify the importance of the particular plant to the community and recorded when there many usereports for the plant while low UVs are recorded when there are few use- reports.

Informant Consensus factor $\left(F_{i c}\right)$ :-

Informant Consensus factor or $\mathrm{F}_{\mathrm{ic}}$ was employed to identify the uniformity of the informants on the reported cures for the group of ailments. This method was based along the Informant Agreement Ratio (IAR) of Trotter and Logan (1986) and consequently known today as Informant Consensus factor. It was calculated using the following formula: $F_{i c}=N u r-N t /(N u r-1)$, where Nur is the number of use citations in each category and $\mathrm{Nt}$ is the number of species used.

$\mathrm{F}_{\mathrm{ic}}$ illustrates the degree of agreement among the informants to the use of a particular plant species and ranges between 0 and 1 . This criterion can effectively sort out interesting plants for the search of novel drugs (Canales $e t$ al., 2005). High $F_{\text {ic }}$ values approaching to 1 was obtained when the documented plants are used by a wide proportion of the informants for a selective disease category while low $F_{i c}$ shows disagreement among the informants which may be due to lack of knowledge sharing (Heinrich et al., 1998; Grazzaneo et al., 2005).

\section{Fidelity Level (FL):-}

The percentage of informants claiming the use of a certain plant for the same major purpose was calculated for the most frequently reported diseases by the Fidelity Level. It was developed by Friedman et al., (1986) and calculated using the following formula:

FL $(\%)=\mathrm{Np} / \mathrm{N} \times 100$, where $\mathrm{Np}$ is the number of informants claiming a use of a plant species to treat a particular disease and $\mathrm{N}$ is the number of informants that use the plants as a medicine to treat any given disease.

Fidelity level are accounted as highest when the value nearly approaches $100 \%$ where the particular plant species are reported as the most preferred for a certain ailment while low FLs denotes the usage of the plants in many different ailments and are least preferred by the informants.

\section{Results And Discussion:-}

The ethnobotanical data collected, identified and documented from various sites of Maring inhabiting areas of Chandel district of Manipur were analyze critically. The study documented 144 medicinal plant species in 66 families categories across 18 ailments. The family Asteraceae was the most represented with 14 plant species followed by Lamiaceae with 10 species, Zingiberaceae, Cucurbitaceae and Verbenaceae with 7 species while Fabaceae was represented with 6 species (Fig. 2).

The most used plant parts was found to be leaves with $54 \%$, Whole plant with $12 \%$, Root $-9 \%$, Fruit- $7 \%$ and Rhizome with $5 \%$ (Fig. 3).

Table 3:-Informant consensus factor of the medicinal plants documented from the Maring tribe of Chandel district

\begin{tabular}{|c|c|c|c|}
\hline Ailments categories & $\begin{array}{c}\text { No. of Use Report } \\
(\text { Nur })\end{array}$ & Number of taxa (Nt) & $\begin{array}{c}\text { Informant } \\
\text { consensus factor } \\
(\text { Fic })\end{array}$ \\
\hline Liver Problem (LP) & 29 & 6 & 0.79 \\
\hline Circulatory System Disorder (CSD) & 126 & 21 & 0.84 \\
\hline
\end{tabular}




\begin{tabular}{|c|c|c|c|}
\hline Antidote (Ad) & 41 & 9 & 0.80 \\
\hline Endocrinal Disorder (ED) & 18 & 6 & 0.73 \\
\hline Respiratory System Disorders (RSD) & 45 & 11 & 0.77 \\
\hline Fever (Fr) & 50 & 15 & 0.71 \\
\hline $\begin{array}{c}\text { Skeleton Muscular System Disorders } \\
\text { (SMSD) }\end{array}$ & 79 & 8 & 0.91 \\
\hline Gastro intestinal disorders (GID) & 54 & 18 & 0.67 \\
\hline Ear, Nose, Throat problem (ENT) & 30 & 13 & 0.58 \\
\hline Dermatological infection (DI) & 43 & 6 & 0.64 \\
\hline Kidney Stone (KS) & 21 & 32 & 0.75 \\
\hline Genito-urinary disorders (GUD) & 176 & 6 & 0.82 \\
\hline Oral Care (OC) & 50 & 5 & 0.89 \\
\hline Oncogenes (Og) & 25 & 5 & 0.71 \\
\hline Piles (P) & 18 & 2 & 0.76 \\
\hline Deworming (Dw) & 4 & 2 & 0.66 \\
\hline Smallpox (Sp) & 5 & 4 & 0.75 \\
\hline General Health (GH) & 14 & $\mathbf{1 8 5}$ & 0.76 \\
\hline Total & $\mathbf{8 2 8}$ & & \\
\hline
\end{tabular}

Table 4:-Number of Use-report (citation), Use-value, mode of administration and other ingredients added for the medicinal plants and herbal therapy used by the Maring

\begin{tabular}{|c|c|c|c|c|c|}
\hline $\begin{array}{l}\text { Scientific Name (with } \\
\text { family) }\end{array}$ & $\begin{array}{l}\text { Parts } \\
\text { used }\end{array}$ & $\begin{array}{l}\text { Ailments category : No. } \\
\text { of use-report }\end{array}$ & $\begin{array}{l}\text { Use- } \\
\text { value } \\
\text { (UV) }\end{array}$ & $\begin{array}{l}\text { Administration } \\
\text { and } \\
\text { preparation }\end{array}$ & $\begin{array}{l}\text { Other } \\
\text { ingredients }\end{array}$ \\
\hline $\begin{array}{l}\text { Achyranthes aspera } \mathrm{L} . \\
\text { (Amaranthaceae) }\end{array}$ & $\mathrm{Lf}$ & $\begin{array}{l}\text { GID:3 (stomachache) } \\
\text { Ad:4 }\end{array}$ & 0.70 & $\begin{array}{l}\text { Oral/topical } \\
\text { (decoction, } \\
\text { paste) }\end{array}$ & - \\
\hline $\begin{array}{l}\text { Acmella paniculata } \\
\text { (Wall. ex DC.) R. K. } \\
\text { Jansen (Asteraceae) }\end{array}$ & $\mathrm{Lf}$ & OC: 8 (tooth cavities) & 0.80 & Oral (paste) & - \\
\hline $\begin{array}{l}\text { Acorus calamus } \mathrm{L} . \\
\text { (Acoraceae) }\end{array}$ & $\mathrm{Rh}$ & Fr:7 (fever) & 0.70 & Inhalation (raw) & - \\
\hline $\begin{array}{l}\text { Aegle marmelos (L.) } \\
\text { Corr. } \\
\text { (Rutaceae) }\end{array}$ & Fr & GID:2 (stomachache) & 0.20 & Oral (Roasted) & - \\
\hline $\begin{array}{l}\text { Adiantum philippense L. } \\
\text { (Adiantaceae) }\end{array}$ & $\mathrm{Wh}$ & ED: 2 (diabetes) & 0.20 & Oral (Decoction) & - \\
\hline $\begin{array}{l}\text { Agave americana } \mathrm{L} . \\
\text { (Asparagaceae) }\end{array}$ & Lf & Fr:1 (fever) RSD:2 (cold) & 0.30 & Oral (Juice) & - \\
\hline $\begin{array}{l}\text { Ageratum conyzoides } \mathrm{L} . \\
\text { (Asteraceae) }\end{array}$ & $\mathrm{Lf}$ & $\begin{array}{l}\text { CSD:6 (blood clot) } \\
\text { GUD: } 4 \text { (pregnant } \\
\text { complicacies) }\end{array}$ & 1 & $\begin{array}{l}\text { Topical } \\
\text { (paste/decoction) }\end{array}$ & Rice water \\
\hline $\begin{array}{l}\text { Allium hookeri } \mathrm{L} . \\
\text { (Amaryllidaceae) }\end{array}$ & Lf & $\begin{array}{l}\text { CSD:7 (blood pressure) } \\
\text { SMSD: } 3 \text { (headache) }\end{array}$ & 1 & $\begin{array}{l}\text { Oral } \\
\text { (steamed/raw) }\end{array}$ & - \\
\hline $\begin{array}{l}\text { Allium tuberosum Roxb. } \\
\text { (Amaryllidaceae) }\end{array}$ & $\mathrm{Lf}$ & GUD:4 (dysuria) & 0.40 & $\begin{array}{l}\text { Oral (decoction/ } \\
\text { raw) }\end{array}$ & - \\
\hline $\begin{array}{l}\text { Alocasia macrorrhiza } \\
\text { Schott. (Araceae) }\end{array}$ & $\mathrm{Cm}$ & DI:3 (burns) & 0.30 & Topical (paste) & - \\
\hline $\begin{array}{l}\text { Aloe vera Mill Gard. } \\
\text { (Aphodelaceae) }\end{array}$ & $\overline{\mathrm{Lf}}$ & $\begin{array}{l}\text { GID: } 2 \text { (stomach ulcer) } \\
\text { DI: } 6 \text { (burns) }\end{array}$ & 0.80 & $\begin{array}{l}\text { Oral/topical } \\
\text { (raw) }\end{array}$ & - \\
\hline $\begin{array}{l}\text { Alpinia galanga Willd. } \\
\text { (Zingiberaceae) }\end{array}$ & $\mathrm{Rh}$ & $\begin{array}{l}\text { ENT : } 2 \text { (sore throat) } \\
\text { RSD: } 4 \text { (cough) }\end{array}$ & 0.60 & Oral (raw) & - \\
\hline $\begin{array}{l}\text { Amaranthus viridis } \mathrm{L} . \\
\text { (Amaranthaceae) }\end{array}$ & Lf & GID: 2 (constipation) & 0.20 & Oral (cooked) & - \\
\hline
\end{tabular}




\begin{tabular}{|c|c|c|c|c|c|}
\hline $\begin{array}{l}\text { Ananas comosus (L.) } \\
\text { Merr. (Bromeliaceae) }\end{array}$ & Lf & $\begin{array}{l}\text { Fr: } 1 \text { (fever) DI: } 2 \text { (skin } \\
\text { infection) }\end{array}$ & 0.30 & $\begin{array}{l}\text { Oral/topical } \\
\text { (Juice/roasted) }\end{array}$ & - \\
\hline $\begin{array}{l}\text { Andrographis paniculata } \\
\text { Nees (Acanthaceae) }\end{array}$ & $\mathrm{Lf}$ & OC: 2 (mouth ulcer) & 0.20 & Oral (decoction) & Sugar candy \\
\hline $\begin{array}{l}\text { Anotis foetida (Dalz.) } \\
\text { Benth. \& Hook.f. } \\
\text { (Rubiaceae) }\end{array}$ & $\mathrm{Rt}$ & $\begin{array}{l}\text { SMSD: } 3 \text { (fracture bone) } \\
\text { DI: } 1 \text { (boils) }\end{array}$ & 0.40 & Topical (paste) & - \\
\hline $\begin{array}{l}\text { Ardisia colorata Roxb. } \\
\text { (Myrsinaceae) }\end{array}$ & $\mathrm{Fr} / \mathrm{Bk} / \mathrm{Lf}$ & $\begin{array}{l}\text { ENT: } 1 \text { (sore throat) } \\
\text { GID: } 2 \text { (dysentery, } \\
\text { diarrhea) }\end{array}$ & 0.30 & $\begin{array}{l}\text { Oral } \\
\text { (decoction/raw) }\end{array}$ & - \\
\hline $\begin{array}{l}\text { Artimisia nilagirica (C.B. } \\
\text { Clarke) Pamp. } \\
\text { (Asteraceae) }\end{array}$ & $\mathrm{Lf}$ & $\begin{array}{l}\text { GUD: } 3 \text { (pregnant } \\
\text { complicacies) }\end{array}$ & 0.30 & $\begin{array}{l}\text { Topical } \\
\text { (decoction) }\end{array}$ & - \\
\hline $\begin{array}{l}\text { Arundo donax } \mathrm{L} . \\
\text { (Poaceae) }\end{array}$ & Sh & $\begin{array}{l}\text { Fr: } 1 \text { (fever) Dw: } 1 \\
\text { (worm expulsion) }\end{array}$ & 0.20 & $\begin{array}{l}\text { Topical/Oral } \\
\text { (paste/decoction) }\end{array}$ & - \\
\hline $\begin{array}{l}\text { Azadirachta indica A. } \\
\text { Juss. (Meliaceae) }\end{array}$ & Lf & $\begin{array}{l}\text { Sp: } 2 \text { (smallpox) Fr: } 3 \\
\text { (fever) ENT: } 1 \text { (sore } \\
\text { throat) }\end{array}$ & 0.60 & $\begin{array}{l}\text { Topical/Oral } \\
\text { (Decoction/raw) }\end{array}$ & - \\
\hline $\begin{array}{l}\text { Bambusa nutans Wall. } \\
\text { (Poaceae) }\end{array}$ & $\mathrm{Sh}$ & Ad: 3 (Dog/snake bites) & 0.30 & Topical (paste) & - \\
\hline $\begin{array}{l}\text { Bauhinia purpurea } \mathrm{L} . \\
\text { (Caesalpiniaceae) }\end{array}$ & $\mathrm{Fl}$ & $\begin{array}{l}\text { GUD: } 2 \text { (menstrual } \\
\text { disorder, leucorrhoea) }\end{array}$ & 0.20 & Oral (paste) & - \\
\hline $\begin{array}{l}\text { Benincasa hispida } \\
\text { (Thunb.) Cogn. } \\
\text { (Cucurbitaceae) }\end{array}$ & $\mathrm{Fr}$ & Ad: 2 (food poisoning) & 0.20 & Oral (raw) & - \\
\hline $\begin{array}{l}\text { Blumeopsis flava (DC.) } \\
\text { Gagnep } \\
\text { (Asteraceae) }\end{array}$ & Lf & ENT: 2 (sinuses) & 0.20 & $\begin{array}{l}\text { Inhalation } \\
\text { (paste/smoked) }\end{array}$ & - \\
\hline $\begin{array}{l}\text { Brugmansia sauveolens } \\
\text { (Humb. \& Bonpl. ex. } \\
\text { Willd.) Bercht. \& J. } \\
\text { Presl. (Solanaceae) }\end{array}$ & Lf & Ad: 5 (snake/dog bites) & 0.50 & Topical (paste) & - \\
\hline $\begin{array}{l}\text { Bryonopsis lociniosa } \\
\text { Naud. } \\
\text { (Cucurbitaceae) }\end{array}$ & $\mathrm{Lf}$ & RSD: 2 (asthma) & 0.20 & $\begin{array}{l}\text { Inhalation } \\
\text { (smoked) }\end{array}$ & - \\
\hline $\begin{array}{l}\text { Butea minor (Lam.) } \\
\text { Kunze. (Fabaceae) }\end{array}$ & $\mathrm{Bk}$ & Ad: 1 (snake/dog bites) & 0.10 & Topical (paste) & - \\
\hline $\begin{array}{l}\text { Cajanus cajan (L.) } \\
\text { Millsp. } \\
\text { (Fabaceae) }\end{array}$ & $\mathrm{Lf} / \mathrm{Rt}$ & Og: 1 (cancer) & 0.10 & Oral (decoction) & - \\
\hline $\begin{array}{l}\text { Cannabis sativa } \mathrm{L} . \\
\text { (Cannabinaceae) }\end{array}$ & Lf & SMSD: 4 (bodyache) & 0.40 & $\begin{array}{l}\text { Topical } \\
\text { (decoction) }\end{array}$ & - \\
\hline $\begin{array}{l}\text { Carica papya } \mathrm{L} . \\
\text { (Caricaceae) }\end{array}$ & Rt & GUD: 2 (leucorrhoea) & 0.20 & Oral (decoction) & Sugar candy \\
\hline $\begin{array}{l}\text { Cassia fistula } \mathrm{L} . \\
\text { (Caesalpiniaceae) }\end{array}$ & $\mathrm{Lf}$ & DI: 1 (skin rashes) & 0.10 & Topical (paste) & - \\
\hline $\begin{array}{l}\text { Celosia argentea } \mathrm{L} . \\
\text { (Amaranthaceae) }\end{array}$ & Lf & CSD: 2 (blood clot) & 0.20 & Topical (paste) & - \\
\hline $\begin{array}{l}\text { Cissus adnata Roxb. } \\
\text { (Vitaceae) }\end{array}$ & Lf & KS: 4 (kidney stone) & 0.40 & Oral (decoction) & - \\
\hline $\begin{array}{l}\text { Cissus discolor Blume } \\
\text { (Vitaceae) }\end{array}$ & Lf & KS: 5 (kidney stone) & 0.50 & Oral (decoction) & - \\
\hline Clerodendrum & Lf & CSD: 4 (blood pressure) & 0.40 & Oral (decoction) & - \\
\hline
\end{tabular}




\begin{tabular}{|c|c|c|c|c|c|}
\hline $\begin{array}{l}\text { colebrookianum Walp. } \\
\text { (Lamiaceae) }\end{array}$ & & & & & \\
\hline $\begin{array}{l}\text { Clerodendrum indicum } \\
\text { (L.) Kuntze (Lamiaceae) }\end{array}$ & $\mathrm{Lf} / \mathrm{Rt}$ & GUD: 1 (Dysuria) & 0.10 & Oral (decoction) & - \\
\hline $\begin{array}{l}\text { Clerodendrum } \\
\text { philippinum Schauer } \\
\text { (Lamiaceae) }\end{array}$ & $\mathrm{Lf}$ & SMSD: 1 (bodyache) & 0.10 & $\begin{array}{l}\text { Topical } \\
\text { (decoction) }\end{array}$ & - \\
\hline $\begin{array}{l}\text { Clerodendrum serratum } \\
\text { Spreng. (Verbenaceae) }\end{array}$ & $\mathrm{Lf}$ & GH: 2 (blood tonic) & 0.20 & Oral (decoction) & - \\
\hline $\begin{array}{l}\text { Coix lachrymal jobi L. } \\
\text { (Poaceae) }\end{array}$ & Lf & GUD: 4 (Dysuria) & 0.40 & Oral (decoction) & - \\
\hline $\begin{array}{l}\text { Costus speciosus } \\
\text { (Koening) Sm. } \\
\text { (Costaceae) }\end{array}$ & $\mathrm{Lf}$ & $\begin{array}{l}\text { Fr: } 2 \text { (fever) CSD: } 2 \\
\text { (blood clot) }\end{array}$ & 0.40 & $\begin{array}{l}\text { Oral/topical } \\
\text { (paste) }\end{array}$ & - \\
\hline $\begin{array}{l}\text { Crassocephalum } \\
\text { crepidioides S. Moore } \\
\text { (Asteraceae) }\end{array}$ & Lf & GID: 2 (stomach ulcer) & 0.20 & Oral (decoction) & - \\
\hline $\begin{array}{l}\text { Crataeva magna (Lour.) } \\
\text { DC. (Capparidaceae) }\end{array}$ & $\mathrm{Lf}$ & SMSD: 5 (bodyache) & 0.50 & $\begin{array}{l}\text { Topical } \\
\text { (decoction) }\end{array}$ & - \\
\hline $\begin{array}{l}\text { Crotolaria juncea } \mathrm{L} . \\
\text { (Fabaceae) }\end{array}$ & $\mathrm{Lf}$ & GH: 2 (blood tonic) & 0.20 & $\begin{array}{l}\text { Oral } \\
\text { (raw/cooked) }\end{array}$ & - \\
\hline $\begin{array}{l}\text { Cucurma angustifolia } \\
\text { Roxb. (Zingiberaceae) }\end{array}$ & $\mathrm{Rh}$ & GID: 2 (indigestion) & 0.20 & Oral (raw) & - \\
\hline $\begin{array}{l}\text { Cucurma caesia Roxb. } \\
\text { (Zingiberaceae) }\end{array}$ & $\mathrm{Rh}$ & GID: 4 (stomachache) & 0.40 & Oral (raw/dried) & - \\
\hline $\begin{array}{l}\text { Cucurma domestica Val. } \\
\text { (Zingiberaceae) }\end{array}$ & $\mathrm{Rh}$ & CSD: 6 (blood clot) & 0.60 & Topical (raw) & - \\
\hline $\begin{array}{l}\text { Cuscuta reflexa Roxb. } \\
\text { (Convolvulaceae) }\end{array}$ & $\mathrm{Wh}$ & $\begin{array}{l}\text { LP: } 5 \text { (jaundice) CSD: } 2 \\
\text { (blood clot) }\end{array}$ & 0.70 & Oral (decoction) & - \\
\hline $\begin{array}{l}\text { Cymbopogon citrates } \\
\text { Stapf (Poaceae) }\end{array}$ & $\mathrm{Lf}$ & Fr: 1 (fever) & 0.10 & Oral (decoction) & - \\
\hline $\begin{array}{l}\text { Cynodon dactylon (L.) } \\
\text { Pers. (Poaceae) }\end{array}$ & Rt & $\begin{array}{l}\text { GUD:3 (Dysuria) GID: } \\
\text { 1(stomachache) }\end{array}$ & 0.40 & Oral (juice) & - \\
\hline $\begin{array}{l}\text { Dalbergia stipulacea } \mathrm{L} . \\
\text { (Fabaceae) }\end{array}$ & $\mathrm{Bk}$ & OC: 9 (tooth cavities) & 0.90 & Topical (juice) & - \\
\hline $\begin{array}{l}\text { Datura stramonium L. } \\
\text { (Solanaceae) }\end{array}$ & Lf & $\begin{array}{l}\text { DI: 1(boils) Ad: } 3 \text { (snake } \\
\text { bites) }\end{array}$ & 0.40 & Topical (paste) & - \\
\hline $\begin{array}{l}\text { Daucus carota } \mathrm{L} . \\
\text { (Apiaceae) }\end{array}$ & $\mathrm{Rt} / \mathrm{Sd}$ & $\begin{array}{l}\text { GUD: } 2 \text { (irregular } \\
\text { menstruation) ENT:3 } \\
\text { (eye infection) }\end{array}$ & 0.50 & $\begin{array}{l}\text { Oral (decoction/ } \\
\text { raw) }\end{array}$ & - \\
\hline $\begin{array}{l}\text { Dicrocephala integrifolia } \\
\text { Kuntz } \\
\text { (Asteraceae) }\end{array}$ & $\mathrm{Lf}$ & $\begin{array}{l}\text { GID: } 1 \text { (indigestion) } \\
\text { GUD: } 2 \text { (labor pain) }\end{array}$ & 0.30 & $\begin{array}{l}\text { Topical/oral } \\
\text { (decoction) }\end{array}$ & - \\
\hline $\begin{array}{l}\text { Dioscorea alata } \mathrm{L} . \\
\text { (Dioscoreaceae) }\end{array}$ & $\mathrm{Tb}$ & $\begin{array}{l}\text { LP: } 1 \text { (jaundice) GID: } 2 \\
\text { (stomachache) }\end{array}$ & 0.30 & Oral (juice) & $\begin{array}{l}\text { Saccharum } \\
\text { officinale, } \\
\text { Centella asiatica, } \\
\text { Cucurma } \\
\text { aromatica and } \\
\text { Jeera }\end{array}$ \\
\hline $\begin{array}{l}\text { Drymaria cordata Willd. } \\
\text { (Caryophyllaceae) }\end{array}$ & $\mathrm{Wh}$ & $\begin{array}{l}\text { RSD: 3(asthma) ENT: } 1 \\
\text { (night blindness) }\end{array}$ & 0.40 & $\begin{array}{l}\text { Inhalation/ oral } \\
\text { (smoked/ } \\
\text { decoction) }\end{array}$ & - \\
\hline Duranta repens L. & Lf & Og: 4 (tumors) & 0.40 & Topical (paste) & - \\
\hline
\end{tabular}




\begin{tabular}{|c|c|c|c|c|c|}
\hline (Verbenaceae) & & & & & \\
\hline $\begin{array}{l}\text { Eclipta prostrata } \mathrm{L} . \\
\text { (Asteraceae) }\end{array}$ & $\mathrm{Lf}$ & Fr: 2 (fever) & 0.20 & Oral (decoction) & Honey \\
\hline $\begin{array}{l}\text { Elsholtzia blanda Benth. } \\
\text { (Lamiaceae) }\end{array}$ & Lf/If & ENT: 2 (tonsilites) & 0.20 & Oral (raw) & - \\
\hline $\begin{array}{l}\text { Elsholtzia communis } \\
\text { (Coll. \& Hemsl.) Diels } \\
\text { (Lamiaceae) }\end{array}$ & $\mathrm{Wh}$ & ENT: 1(tonsilites) & 0.10 & Oral (decoction) & - \\
\hline $\begin{array}{l}\text { Enhydra fluctuans Lour. } \\
\text { (Asteraceae) }\end{array}$ & Sh & $\begin{array}{l}\text { ED: } 5 \text { (diabetes) GUD: } 2 \\
\text { (leucorrhoea) }\end{array}$ & 0.70 & Oral (decoction) & Sugar candy \\
\hline $\begin{array}{l}\text { Entada pursaetha DC. } \\
\text { (Mimosaceae) }\end{array}$ & Lf & ED: 1 (diabetes) & 0.10 & Oral (decoction) & - \\
\hline $\begin{array}{l}\text { Equisetum ramosissimum } \\
\text { Desf. ssp. Debile (Roxb.) } \\
\text { Hauhe } \\
\text { (Equisetaceae) }\end{array}$ & $\mathrm{Wh}$ & ENT: 2 (epistaxis) & 0.20 & $\begin{array}{l}\text { Inhalation } \\
\text { (smoked) }\end{array}$ & - \\
\hline $\begin{array}{l}\text { Eryngium foetidum } \\
\text { L.(Apiaceae) }\end{array}$ & $\mathrm{Wh}$ & CSD: 1 (blood pressure) & 0.10 & $\begin{array}{l}\text { Oral/inhalation } \\
\text { (decoction/paste) }\end{array}$ & - \\
\hline $\begin{array}{l}\text { Eupatorium } \\
\text { adenophorum Spreng. } \\
\text { (Asteraceae) }\end{array}$ & $\mathrm{Lf}$ & CSD: 3 (blood clot) & 0.30 & Topical (paste) & - \\
\hline $\begin{array}{l}\text { Eupatorium birmanicum } \\
\text { DC. (Asteraceae) }\end{array}$ & Lf & $\begin{array}{l}\text { GID: } 1 \text { (stomach ulcer) } \\
\text { OC: } 3 \text { (mouth ulcer) }\end{array}$ & 0.40 & Oral (decoction) & - \\
\hline $\begin{array}{l}\text { Eupatorium odoratum L. } \\
\text { (Asteraceae) }\end{array}$ & Lf & CSD: 4 (blood clot) & 0.40 & Topical (paste) & - \\
\hline $\begin{array}{l}\text { Euphorbia hirta } \mathrm{L} . \\
\text { (Euphorbiaceae) }\end{array}$ & $\mathrm{Wh}$ & GUD: 1 (leucorrhoea) & 0.10 & Oral (decoction) & Prawn \\
\hline $\begin{array}{l}\text { Ficus assamica Miq. } \\
\text { (Moraceae) }\end{array}$ & Rt & GUD: 1 (leucorrhoea) & 0.10 & Oral (decoction) & - \\
\hline $\begin{array}{l}\text { Ficus benghalensis L. } \\
\text { (Moraceae) }\end{array}$ & $\mathrm{Bk}$ & $\begin{array}{l}\text { GUD: } 3 \text { (irregular } \\
\text { menstruation) }\end{array}$ & 0.30 & Oral (paste) & - \\
\hline $\begin{array}{l}\text { Ficus hispida L.f. } \\
\text { (Moraceae) }\end{array}$ & $\mathrm{Lf}$ & ED: 1 (diabetes) & 0.10 & Oral (decoction) & - \\
\hline $\begin{array}{l}\text { Fragaria nillgerensis } \\
\text { Schlecht. ex. J. Gay } \\
\text { (Rosaceae) }\end{array}$ & $\mathrm{Lf}$ & GUD: 5 (Dysuria) & 0.50 & Oral (decoction) & - \\
\hline $\begin{array}{l}\text { Garcinia pedunculata } \\
\text { Roxb. ex Buch. } \\
\text { (Clusiaceae) }\end{array}$ & $\mathrm{Fr}$ & $\begin{array}{l}\text { ENT: } 1 \text { (sore throat) } \\
\text { RSD: } 2 \text { (cough) }\end{array}$ & 0.30 & Oral (boiled) & - \\
\hline $\begin{array}{l}\text { Glochidion coccineum } \\
\text { Forst (Phyllanthaceae) }\end{array}$ & $\mathrm{Lf}$ & CSD: 3 (blood clot) & 0.30 & Topical (paste) & - \\
\hline $\begin{array}{l}\text { Gmelina arborea Roxb. } \\
\text { (Verbenaceae) }\end{array}$ & $\mathrm{Lf}$ & Ad: 3 (snake bites) & 0.30 & Topical (paste) & - \\
\hline $\begin{array}{l}\text { Hedychium greenii } \\
\text { Smith. } \\
\text { (Zingiberaceae) }\end{array}$ & $\mathrm{Rt}$ & GUD: 1 (leucorrhoea) & 0.10 & Oral (decoction) & - \\
\hline $\begin{array}{l}\text { Hedychium spicatum Rao } \\
\text { \& Verma (Zingiberaceae) }\end{array}$ & $\mathrm{Rh}$ & GUD: 1 (leucorrhoea) & 0.10 & Oral (decoction) & - \\
\hline $\begin{array}{l}\text { Hibiscus sabdariffa } \mathrm{L} \text {. } \\
\text { (Malvaceae) }\end{array}$ & $\mathrm{Lf} / \mathrm{Sc}$ & $\begin{array}{l}\text { CSD:2 (blood } \\
\text { purification) GUD: } 3 \\
\text { (Pregnant complicacies) }\end{array}$ & 0.50 & $\begin{array}{l}\text { Topical/oral } \\
\text { (decoction/raw) }\end{array}$ & - \\
\hline $\begin{array}{l}\text { Holmskioldia sanguinea } \\
\text { Retz. (Verbenaceae) }\end{array}$ & $\mathrm{Lf}$ & $\begin{array}{l}\text { GUD: } 1 \text { (irregular } \\
\text { menstruation) }\end{array}$ & 0.10 & Oral (decoction) & - \\
\hline $\begin{array}{l}\text { Houttuynia cordata } \\
\text { Thunb. (Sauraceae) }\end{array}$ & Wh & ENT: 4 (tonsillitis) & 0.40 & Oral (decoction) & - \\
\hline
\end{tabular}




\begin{tabular}{|c|c|c|c|c|c|}
\hline $\begin{array}{l}\text { Hydrocotyl javanica } \\
\text { Molkenboer ex. C.B. } \\
\text { Clarke (Apiaceae) }\end{array}$ & $\mathrm{Lf}$ & GUD: 1 (Dysuria) & 0.10 & Oral (decoction) & - \\
\hline $\begin{array}{l}\text { Impatiens balsamina } \mathrm{L} . \\
\text { (Balsaminaceae) }\end{array}$ & $\mathrm{Lf}$ & $\begin{array}{l}\text { CSD: } 1 \text { (blood clot) DI: } 2 \\
\text { (skin infection) }\end{array}$ & 0.30 & Topical (paste) & - \\
\hline $\begin{array}{l}\text { Justicia adhatoda } \mathrm{L} . \\
\text { (Acanthaceae) }\end{array}$ & $\mathrm{Lf}$ & $\begin{array}{l}\text { RSD: } 8 \text { (bronchitis) Fr: } 3 \\
\text { (fever) }\end{array}$ & 1.1 & Oral (decoction) & $\begin{array}{l}\text { Zingiber } \\
\text { officinale }\end{array}$ \\
\hline $\begin{array}{l}\text { Justicia gendarussa N.L. } \\
\text { Burman (Acanthaceae) }\end{array}$ & $\mathrm{Lf}$ & SMSD: 8 (bodyache) & 0.80 & $\begin{array}{l}\text { Topical } \\
\text { (decoction) }\end{array}$ & - \\
\hline $\begin{array}{l}\text { Lagenaria siceraria } \\
\text { (Molina) Standl. } \\
\text { (Cucurbitaceae) }\end{array}$ & Fr & $\begin{array}{l}\text { KS: } 3 \text { (kidney stone) Ad: } \\
1 \text { (bee sting) }\end{array}$ & 0.40 & Oral (juice) & - \\
\hline $\begin{array}{l}\text { Lantana camara } \mathrm{L} . \\
\text { (Verbenaceae) }\end{array}$ & $\mathrm{Wh}$ & CSD: 5 (blood clot) & 0.50 & Topical (paste) & - \\
\hline $\begin{array}{l}\text { Lindernia ruellioides } \\
\text { (Colsm.) Pennell } \\
\text { (Linderniaceae) }\end{array}$ & $\mathrm{Wh}$ & $\begin{array}{l}\text { KS: } 1 \text { (kidney stone) } \\
\text { GUD: } 9 \text { (Dysuria) }\end{array}$ & 1 & Oral (decoction) & - \\
\hline $\begin{array}{l}\text { Litsea monopetala } \\
\text { (Roxb.) Pers. (Lauraceae) }\end{array}$ & $\mathrm{Rt}$ & $\begin{array}{l}\text { SMSD: } 5 \text { (bodyache) } \\
\text { RSD: } 2 \text { (cough) }\end{array}$ & 0.70 & Topical (paste) & $\begin{array}{l}\text { Animal oils } \\
\text { (preferably pig) }\end{array}$ \\
\hline $\begin{array}{l}\text { Luffa cylindrical } \\
\text { (L.)Roem. } \\
\text { (Cucurbitaceae) }\end{array}$ & $\mathrm{Lf}$ & DI: 5 (burns) & 0.50 & Topical (paste) & - \\
\hline $\begin{array}{l}\text { Lygodium flexuosum (L.) } \\
\text { Sw. (Lygodiaceae) }\end{array}$ & $\mathrm{Wh}$ & LP: 3 (jaundice) & 0.30 & $\begin{array}{l}\text { Oral/topical } \\
\text { (decoction) }\end{array}$ & - \\
\hline $\begin{array}{l}\text { Lysimachia parvifolia } \\
\text { Baker (Primulaceae) }\end{array}$ & Wh & P: 4 (piles) & 0.40 & Oral (boiled) & \\
\hline $\begin{array}{l}\text { Melastoma } \\
\text { malabathricum } \mathrm{L} . \\
\text { (Melastomaceae) }\end{array}$ & $\mathrm{Rt}$ & GUD: 2 (leucorrhoea) & 0.20 & Oral (decoction) & Sugar candy \\
\hline $\begin{array}{l}\text { Mentha spicata } \mathrm{L} . \\
\text { (Lamiaceae) }\end{array}$ & $\mathrm{Lf}$ & GID: 3 (stomach ulcer) & 0.30 & Oral (juice) & - \\
\hline $\begin{array}{l}\text { Messua ferrea } \mathrm{L} . \\
\text { (Clusiaceae) }\end{array}$ & Lf & Fr: 1 (fever) & 0.10 & Oral (juice) & - \\
\hline $\begin{array}{l}\text { Meyna spinosa Robyns. } \\
\text { (Rubiaceae) }\end{array}$ & $\mathrm{Sd}$ & Dw: 3 (worm expulsion) & 0.30 & Topical (paste) & - \\
\hline $\begin{array}{l}\text { Mikania micrantha } \\
\text { Kunth. (Asteraceae) }\end{array}$ & $\mathrm{Lf}$ & $\begin{array}{l}\text { Fr: } 1 \text { (fever) CSD: } 3 \\
\text { (blood clot) }\end{array}$ & 0.40 & $\begin{array}{l}\text { Oral/topical } \\
\text { (decoction/paste) }\end{array}$ & - \\
\hline $\begin{array}{l}\text { Mimosa pudica } \mathrm{L} . \\
\text { (Fabaceae) }\end{array}$ & $\mathrm{Rt}$ & GID: 3 (dysentery) & 0.30 & Oral (decoction) & - \\
\hline $\begin{array}{l}\text { Momordica charantia L. } \\
\text { (Cucurbitaceae) }\end{array}$ & $\mathrm{Lf}$ & Fr: 2 (fever) & 0.20 & Oral (decoction) & - \\
\hline $\begin{array}{l}\text { Mucuna nigricans } \\
\text { (Lour.) Steud. (Fabaceae) }\end{array}$ & $\mathrm{Sd}$ & DI: 1 (boils) & 0.10 & Topical (paste) & - \\
\hline $\begin{array}{l}\text { Musa paradisiaca } \mathrm{L} . \\
\text { (Musaceae) }\end{array}$ & $\mathrm{Rt}$ & $\begin{array}{l}\text { GUD: } 4 \text { (Pregnant } \\
\text { complicacies) }\end{array}$ & 0.40 & Oral (decoction) & Red sugar \\
\hline $\begin{array}{l}\text { Mussaenda glabra Vahl. } \\
\text { (Rubiaceae) }\end{array}$ & $\mathrm{Lf}$ & DI: 2 (skin rashes) & 0.20 & Topical (boiled) & - \\
\hline $\begin{array}{l}\text { Neptunia prostrataBail. } \\
\text { (Mimosaceae) }\end{array}$ & $\mathrm{Wh}$ & ENT: 3 (earache) & 0.30 & $\begin{array}{l}\text { Topical } \\
\text { (decoction) }\end{array}$ & - \\
\hline $\begin{array}{l}\text { Nerium indicum Mill. } \\
\text { (Apocynaceae) }\end{array}$ & Rt & GUD: 2 (abortifacient) & 0.20 & Oral (decoction) & - \\
\hline $\begin{array}{l}\text { Ocimum basilicum } \mathrm{L} . \\
\text { (Lamiaceae) }\end{array}$ & $\mathrm{Wh}$ & CSD: 1 (blood pressure) & 0.10 & $\begin{array}{l}\text { Oral } \\
\text { (raw/steamed) }\end{array}$ & - \\
\hline $\begin{array}{l}\text { Ocimum gratissimum } \mathrm{L} . \\
\text { (Lamiaceae) }\end{array}$ & $\mathrm{Lf}$ & LP: 1 (jaundice) & 0.10 & Oral (juice) & Honey \\
\hline
\end{tabular}




\begin{tabular}{|c|c|c|c|c|c|}
\hline $\begin{array}{l}\text { Ocimum sanctum } \mathrm{L} . \\
\text { (Lamiaceae) }\end{array}$ & Lf & $\begin{array}{l}\text { RSD: } 2 \text { (asthma) DI: } 1 \\
\text { (skin problem) }\end{array}$ & 0.30 & $\begin{array}{l}\text { Oral/topical } \\
\text { (decoction/paste) }\end{array}$ & - \\
\hline $\begin{array}{l}\text { Opuntia stricta (Haw.) } \\
\text { Haw. var. dillenii (Ker- } \\
\text { Gawler) Benson } \\
\text { (Cactaceae) }\end{array}$ & $\mathrm{Rt}$ & GUD: 2 (leucorrhoea) & 0.20 & Oral (decoction) & Sugar candy \\
\hline $\begin{array}{l}\text { Oroxylum indicum Vent. } \\
\text { (Bignoniaceae) }\end{array}$ & $\mathrm{Fr}$ & $\begin{array}{l}\text { RSD: } 3 \text { (tuberculosis) } \\
\text { Og: } 6 \text { (cancer) }\end{array}$ & 0.90 & Oral (roasted) & - \\
\hline $\begin{array}{l}\text { Oxalis corniculata } \mathrm{L} . \\
\text { (Oxalidaceae) }\end{array}$ & $\mathrm{Wh}$ & $\begin{array}{l}\text { LP: } 4 \text { (jaundice) GUD: } 3 \\
\text { (pregnant complicacies) }\end{array}$ & 0.70 & $\begin{array}{l}\text { Oral/topical } \\
\text { (decoction) }\end{array}$ & Salt \\
\hline $\begin{array}{l}\text { Paederia foetida } \mathrm{L} . \\
\text { (Rubiaceae) }\end{array}$ & $\overline{\mathrm{Lf}}$ & $\begin{array}{l}\text { CSD: } 2 \text { (high blood } \\
\text { pressure) RSD: } 2 \\
\text { (tuberculosis) }\end{array}$ & 0.40 & Oral (decoction) & - \\
\hline $\begin{array}{l}\text { Passiflora edulis Sims. } \\
\text { (Passifloraceae) }\end{array}$ & $\mathrm{Lf}$ & ED: 2 (diabetes) & 0.20 & Oral (decoction) & - \\
\hline $\begin{array}{l}\text { Phaseolus lunatus } \\
\text { L.(Fabaceae) }\end{array}$ & $\mathrm{Lf}$ & Fr: 2 (fever) & 0.20 & Topical (paste) & $\begin{array}{l}\text { Mustard } \\
\text { oil/kerosene }\end{array}$ \\
\hline $\begin{array}{l}\text { Phlogacanthus } \\
\text { thyrsiformis (Roxb. ex. } \\
\text { Hardw.) Mabb. } \\
\text { (Acanthaceae) }\end{array}$ & LF & $\begin{array}{l}\text { Fr: } 5 \text { (fever) Sp: } 5 \\
\text { (smallpox) }\end{array}$ & 1 & Oral (decoction) & - \\
\hline $\begin{array}{l}\text { Pholidota articulata } \\
\text { Lindley (Orchidaceae) }\end{array}$ & $\mathrm{St}$ & CSD: 1 (blood clot) & 0.10 & Topical (paste) & - \\
\hline $\begin{array}{l}\text { Phyllanthus urinaria } \mathrm{L} . \\
\text { (Euphorbiaceae) }\end{array}$ & $\mathrm{Wh}$ & $\begin{array}{l}\text { ED: } 2 \text { (diabetes) DI: } 1 \\
\text { (skin infection) }\end{array}$ & 0.30 & $\begin{array}{l}\text { Oral/topical } \\
\text { (decoction/paste) }\end{array}$ & - \\
\hline $\begin{array}{l}\text { Pinus kesiya Royle } \\
\text { (Pinaceae) }\end{array}$ & St & $\begin{array}{l}\text { GUD: } 3 \text { (pregnant } \\
\text { complicacies) DI: } 2 \\
\text { (ringworm) }\end{array}$ & 0.50 & $\begin{array}{l}\text { Topical } \\
\text { (resin/decoction) }\end{array}$ & - \\
\hline $\begin{array}{l}\text { Piper longum L. } \\
\text { (Piperaceae) }\end{array}$ & $\mathrm{Sd}$ & $\begin{array}{l}\text { GUD: } 2 \text { (menstrual } \\
\text { disorder) }\end{array}$ & 0.20 & Oral (decoction) & - \\
\hline $\begin{array}{l}\text { Plantago erosa Wall. } \\
\text { (Plantaginaceae) }\end{array}$ & $\mathrm{Lf}$ & CSD: 1 (blood clot) & 0.10 & Topical (paste) & - \\
\hline $\begin{array}{l}\text { Plumbago zeylanica } \mathrm{L} \text {. } \\
\text { (Plumbaginaceae) }\end{array}$ & $\mathrm{Lf}$ & Fr: 3 (fever) & 0.30 & Oral (decoction) & - \\
\hline $\begin{array}{l}\text { Polygonum chinense L. } \\
\text { (Polygonaceae) }\end{array}$ & $\mathrm{Lf}$ & KS: 1 (kidney stone) & 0.10 & Oral (decoction) & - \\
\hline $\begin{array}{l}\text { Polygonum plebeium } \\
\text { R.Br. var. plebeium } \mathrm{R} . \mathrm{Br} \text {. } \\
\text { (Apocynaceae) }\end{array}$ & $\mathrm{Lf}$ & GUD: 1 (Dysuria) & 0.10 & Oral (decoction) & - \\
\hline $\begin{array}{l}\text { Polygonum posumba } \\
\text { Buch. Ham. ex. D. Don. } \\
\text { (Polygonaceae) }\end{array}$ & $\mathrm{Lf}$ & $\begin{array}{l}\text { CSD: } 3 \text { (blood pressure) } \\
\text { P: } 5 \text { (piles) }\end{array}$ & 0.80 & $\begin{array}{l}\text { Oral } \\
\text { (raw/cooked) }\end{array}$ & - \\
\hline $\begin{array}{l}\text { Portulaca oleracea } \mathrm{L} . \\
\text { (Portulacaceae) }\end{array}$ & $\mathrm{Wh}$ & $\begin{array}{l}\text { GUD: } 3 \text { (pregnant } \\
\text { complicacies) }\end{array}$ & 0.30 & $\begin{array}{l}\text { Topical } \\
\text { (decoction) }\end{array}$ & - \\
\hline $\begin{array}{l}\text { Potentialla canadensis L. } \\
\text { (Rosaceae) }\end{array}$ & $\mathrm{Lf}$ & KS: 5 (kidney stone) & 0.50 & Oral (decoction) & - \\
\hline $\begin{array}{l}\text { Psidium guajava } \mathrm{L} . \\
\text { (Myrtaceae) }\end{array}$ & $\mathrm{Lf}$ & GID: 5 (dysentery) & 0.50 & Oral (raw) & - \\
\hline $\begin{array}{l}\text { Ricinus communis L. } \\
\text { (Euphorbiaceae) }\end{array}$ & $\mathrm{Lf}$ & CSD: 4 (blood clot) & 0.40 & Topical (paste) & - \\
\hline $\begin{array}{l}\text { Sapindus emarginatus } \\
\text { Vahl. } \\
\text { (Sapindaceae) }\end{array}$ & $\mathrm{Fr}$ & GID: 2 (intestinal worm) & 0.20 & Topical (soaked) & - \\
\hline $\begin{array}{l}\text { Schima wallichii Choisy } \\
\text { (Theaceae) }\end{array}$ & $\mathrm{Lf}$ & GUD: 3 (Dysuria) & 0.30 & Oral (decoction) & - \\
\hline
\end{tabular}




\begin{tabular}{|c|c|c|c|c|c|}
\hline $\begin{array}{l}\text { Scoparia dulcis L. } \\
\text { (Scrophulariaceae) }\end{array}$ & Lf & ENT: 5 (epistaxis) & 0.50 & Oral (juice) & Honey \\
\hline $\begin{array}{l}\text { Scutellaria discolor } \\
\text { Colebr. (Lamiaceae) }\end{array}$ & $\mathrm{Lf}$ & $\begin{array}{l}\text { P: } 1 \text { (piles) GUD: } 1 \\
\text { (menstrual problem) }\end{array}$ & 0.20 & Oral (decoction) & - \\
\hline $\begin{array}{l}\text { Sida rhombifolia } \mathrm{L} . \\
\text { (Malvaceae) }\end{array}$ & $\mathrm{Wh}$ & GUD: 2 (Dysuria) & 0.20 & Oral (decoction) & - \\
\hline $\begin{array}{l}\text { Solanum anguivi Lam. } \\
\text { (Solanaceae) }\end{array}$ & $\mathrm{Fr}$ & RSD: 3 (cough/cold) & 0.30 & Oral (decoction) & - \\
\hline $\begin{array}{l}\text { Solanum torvum } \mathrm{Sw} . \\
\text { (Solanaceae) }\end{array}$ & $\mathrm{Fr}$ & OC: 4 (tooth cavities) & 0.40 & $\begin{array}{l}\text { Topical } \\
\text { (smoked) }\end{array}$ & - \\
\hline $\begin{array}{l}\text { Tamarindus indica } \mathrm{L} . \\
\text { (Caesalpinaceae) }\end{array}$ & $\mathrm{Sd}$ & Ad: 4 (snake/dog bites) & 0.40 & Topical (paste) & - \\
\hline $\begin{array}{l}\text { Tectona grandis } \mathrm{L} . \\
\text { (Verbenaceae) }\end{array}$ & $\mathrm{Lf}$ & $\begin{array}{l}\text { DI: } 1 \text { (ringworm) GH: } 3 \\
\text { (blood tonic) }\end{array}$ & 0.40 & Oral (decoction) & - \\
\hline $\begin{array}{l}\text { Terminalia arjuna } \\
\text { (Roxb.) Wight and Arn. } \\
\text { (Combretaceae) }\end{array}$ & $\mathrm{Bk}$ & GID: 2 (dysentery) & 0.20 & Oral (decoction) & - \\
\hline $\begin{array}{l}\text { Terminalia citrina Roxb. } \\
\text { (Combretaceae) }\end{array}$ & Fr & OC: 3 (mouth ulcer) & 0.30 & Oral (raw) & - \\
\hline $\begin{array}{l}\text { Toona cilliata M. Roem. } \\
\text { (Meliaceae) }\end{array}$ & $\mathrm{Lf}$ & SMSD: 4 (headache) & 0.40 & Oral (juice) & - \\
\hline $\begin{array}{l}\text { Trichosanthes bracteata } \\
\text { (Lam.) Voigt. } \\
\text { (Cucurbitaceae) }\end{array}$ & Fr & Og: 8 (dermal tumor) & 0.80 & Topical (paste) & - \\
\hline $\begin{array}{l}\text { Vitex trifolia } \mathrm{L} . \\
\text { (Verbenaceae) }\end{array}$ & $\mathrm{Wh}$ & DI: 2 (skin rashes) & 0.20 & $\begin{array}{l}\text { Topical } \\
\text { (decoction) }\end{array}$ & - \\
\hline $\begin{array}{l}\text { Xanthium strumarium L. } \\
\text { (Asteraceae) }\end{array}$ & $\mathrm{Lf}$ & Og: 4 (dermal tumor) & 0.40 & $\begin{array}{l}\text { Topical } \\
\text { (decoction) }\end{array}$ & $\begin{array}{l}\text { Oroxylum } \\
\text { indicum, } \\
\text { Drymaria } \\
\text { quercifolia, } \\
\text { Schefflera elata, } \\
\text { Artemisia } \\
\text { parviflora, } \\
\text { Mangifera indica }\end{array}$ \\
\hline $\begin{array}{l}\text { Xanthosoma violaceum } \\
\text { Schott } \\
\text { (Araceae) }\end{array}$ & $\mathrm{St}$ & GH:2 (blood tonic) & 0.20 & Oral (decoction) & - \\
\hline $\begin{array}{l}\text { Xylosma longifolia Clos. } \\
\text { (Flacourtiaceae) }\end{array}$ & $\mathrm{Lf}$ & P: 1 (piles) & 0.10 & Oral (decoction) & - \\
\hline $\begin{array}{l}\text { Zanthoxylum } \\
\text { acanthopodium DC. } \\
\text { (Rutaceae) }\end{array}$ & $\mathrm{Lf}$ & DI: 3 (boils) & 0.30 & Topical (paste) & $\begin{array}{l}\text { Coriandrum } \\
\text { sativum }\end{array}$ \\
\hline $\begin{array}{l}\text { Zehneria scabra (L.f.) } \\
\text { Sonder (Cucurbitaceae) }\end{array}$ & $\mathrm{Lf}$ & LP: 4 (jaundice) & 0.40 & Oral (decoction) & $\begin{array}{l}\text { Imperata } \\
\text { cylindrica }\end{array}$ \\
\hline $\begin{array}{l}\text { Zingiber montanum Link. } \\
\text { ex. A. Dietr. } \\
\text { (Zingiberaceae) }\end{array}$ & $\mathrm{Rh}$ & P: 3 (piles) & 0.30 & Oral (ground) & $\begin{array}{l}\text { Centella asiatica, } \\
\text { Scutellaria } \\
\text { discolor }\end{array}$ \\
\hline
\end{tabular}

Lf: Leaf; Rh: Rhizome; Fr: Fruit; Wh: Whole plant; Cm: Corm; Rt: Root; Bk: Bark; Sh: Shoot; Fl: Flower; Sd: Seed; Tb: Tuber; If: Inflorescence; St: Stem. 
Table 11:-Fidelity level (FL) values of some medicinal plants as claimed by the Maring healers against different ailments.

\begin{tabular}{|c|c|c|c|c|}
\hline Ailment category & Most preferred species & $\mathbf{N}_{\mathbf{P}}$ & $\mathbf{N}$ & $L(\%)$ \\
\hline Liver Problem & $\begin{array}{l}\text { Cuscuta reflexa (Jaundice) } \\
\text { Oxaliscorniculata (Jaundice) }\end{array}$ & $\begin{array}{l}5 \\
6\end{array}$ & $\begin{array}{c}9 \\
12\end{array}$ & $\begin{array}{c}55.55 \\
50\end{array}$ \\
\hline $\begin{array}{l}\text { Circulatory System } \\
\text { Disorder }\end{array}$ & $\begin{array}{l}\text { Ageratum conyzoides (Blood clot) } \\
\text { Allium hookeri (Blood pressure) } \\
\text { Curcuma domestica (Blood clot) }\end{array}$ & $\begin{array}{l}12 \\
14 \\
12\end{array}$ & $\begin{array}{l}20 \\
21 \\
20\end{array}$ & $\begin{array}{c}60 \\
66.66 \\
60\end{array}$ \\
\hline Endocrinal Disorder & Enhydra fluctuans (Diabetes) & 7 & 12 & 58.33 \\
\hline $\begin{array}{l}\text { Respiratory System } \\
\text { Disorders }\end{array}$ & Justicia adhatoda (Bronchitis) & 8 & 11 & 72.72 \\
\hline Fever & $\begin{array}{c}\text { Acorus calamus } \\
\text { Phlogacanthus thyrsiformis }\end{array}$ & $\begin{array}{l}9 \\
6 \\
\end{array}$ & $\begin{array}{c}20 \\
9 \\
\end{array}$ & $\begin{array}{c}45 \\
66.66 \\
\end{array}$ \\
\hline $\begin{array}{c}\text { Skeleton Muscular System } \\
\text { Disorders }\end{array}$ & $\begin{array}{c}\text { Justicia gendarussa (Bodyache) } \\
\text { Litsea monopetala (Bodyache) }\end{array}$ & $\begin{array}{l}18 \\
10\end{array}$ & $\begin{array}{l}20 \\
14 \\
\end{array}$ & $\begin{array}{c}90 \\
71.42 \\
\end{array}$ \\
\hline Gastro intestinal disorders & $\begin{array}{c}\text { Psidium guajava (Dysentery) } \\
\text { Cucurma caesia (Stomachache) }\end{array}$ & $\begin{array}{l}6 \\
6\end{array}$ & $\begin{array}{l}20 \\
20\end{array}$ & $\begin{array}{l}30 \\
30\end{array}$ \\
\hline Dermatological infection & Aloe vera (Burns) & 6 & 8 & 75 \\
\hline Kidney Stone & Cissus discolor & 6 & 20 & 30 \\
\hline Genito-urinary disorders & $\begin{array}{c}\text { Fragaria nillgerensis (Dysuria) } \\
\text { Lindernia ruellioides (Dysuria) } \\
\text { Musa paradisiaca (Pregnant complaints) }\end{array}$ & $\begin{array}{c}10 \\
18 \\
8\end{array}$ & $\begin{array}{l}20 \\
20 \\
20\end{array}$ & $\begin{array}{l}50 \\
90 \\
40\end{array}$ \\
\hline Oral Care & $\begin{array}{c}\text { Acmella paniculata } \\
\text { (Tooth cavities) } \\
\text { Dalbergia stipulacea } \\
\text { (Tooth cavities) }\end{array}$ & $\begin{array}{c}8 \\
18\end{array}$ & $\begin{array}{l}20 \\
20\end{array}$ & $\begin{array}{l}40 \\
90\end{array}$ \\
\hline Oncogenes & $\begin{array}{l}\text { Trichosanthes bracteata } \\
\text { (Dermal tumor) }\end{array}$ & 16 & 20 & 80 \\
\hline Piles & Polygonum posumba & 4 & 10 & 40 \\
\hline Smallpox & Phlogacanthus thyrsiformis & 3 & 9 & 33.33 \\
\hline
\end{tabular}

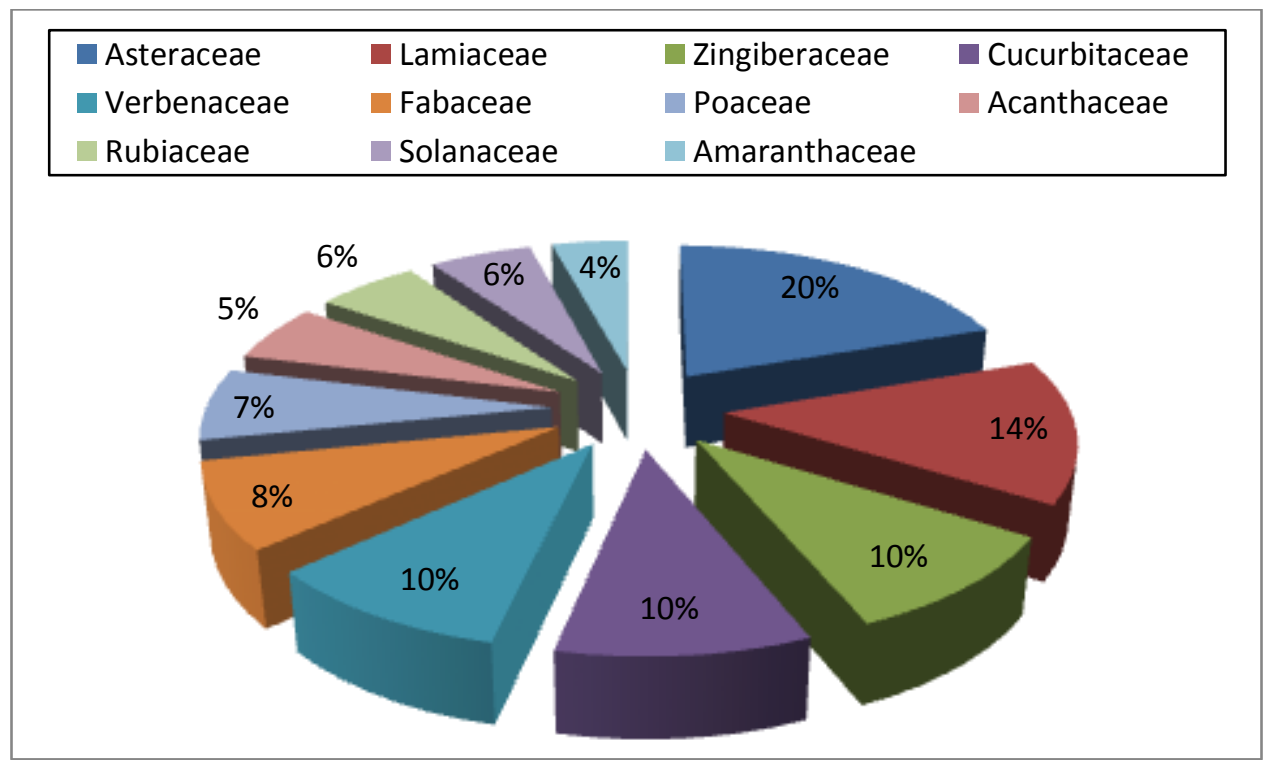

Fig 2:-Percentage of dominant families documented from the study sites. 


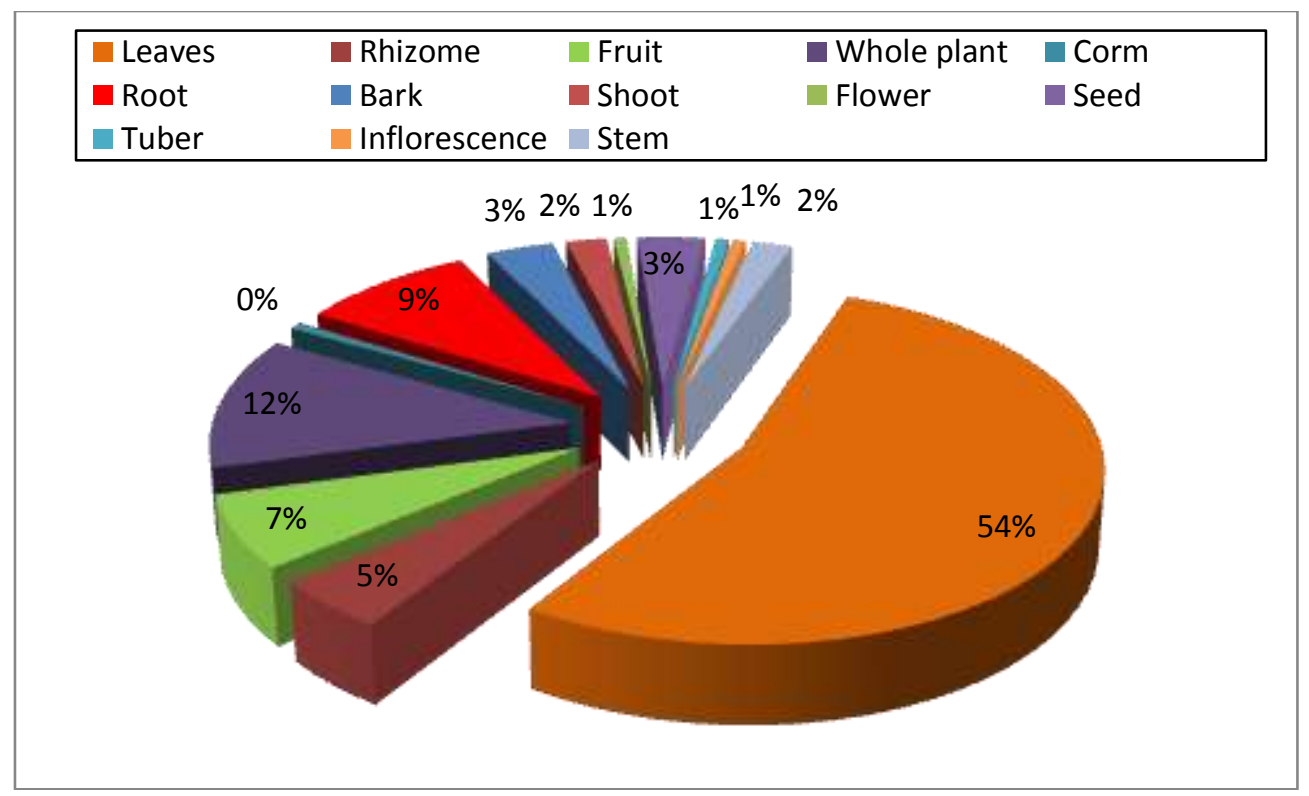

Fig 3:- Percentage of plant parts used by the Maring tribe as medicine

The Informant Consensus Factor or Fic values ranges from 0.91 to 0.58 in the present findings. Skeleton muscular system disorder (SMSD), Oral care (OC) and Circulatory system disorder (CSD) had the highest Fic of 0.91, 0.89 and 0.84 respectively. Higher the value of Fic (close to 1) higher the degree of agreement between the informants of the selected taxa to be used in treatment within a category of ailments while low Fic represents disagreement among the informants (Ragupathy et al., 2008). The highest use reports were recorded from Genito-urinary disorders (GUD) with 176 use reports \& 32 plant species, Circulatory system disorder (CSD) 126 use report \& 21 species, Skeleton muscular system disorder (SMSD) with 79 use report, 8 species. This indicates that there is a good amount of knowledge sharing among the inhabitants regarding the use of plants in the treatment of these ailments. The least agreements among the informant was found in Ear, Nose, Throat (ENT) with Fic of 0.58 followed by Dermatological infection (DI) with a Fic value of 0.64. Dermatological infection had low Fic but this ailment category was ranked fourth and seventh in the number of taxa attributed to the category and number of use report respectively. These indicts that there is lack of knowledge sharing and miscommunication among the informants of the study area on the treatment of these particular ailment categories (Rokaya et al., 2010).

Highest use value was recorded from Allium hookeri L. with 14 use reports by 20 informants giving the value of 1.05. Allium hookeri L. has been well recognized as blood pressure controlling plant in the study areas and also in the treatment of various other diseases, consuming in different form of either raw or cooked. It is included in one of the compulsory plant to be grown in kitchen garden of the Maring tribal community. Other plants with high use value were Ageratum conyzoides L. with 12 use reports by 20 informants giving the value of 1 , Lindernia ruellioides (Colsm.) Pennell with 18 use reports by 20 informants, Dalbergia stipulacea Roxb. with 18 use reports by 20 informants giving value of 0.90 and Justicia gendarussa N.L. Burman with 18 use reports by 20 informants. The major agreements within the ailments categories highlighting the most important species were listed in the table 10.

Lowest use value was recorded from Cymbopogon citrates Stapf which is reported by only one informant with a UV of 0.05 and the particular informant was regularly using this plant during fever. This may be attributed to the widespread use of this plant as health drink as in tea leaf which divert attention on its marketability rather than its medicinal potentiality. The plants with low UV documented in the present study ( 2 use reports by 20 informants) were Arundo donax L., Blumeopsis flava (DC.) Gagnep, Cajanus cajan (L.) Millsp., Crassocephalum crepidioides S. Moore, Curcuma angustifolia Roxb., Elsholtzia blanda Benth., Eryngium foetidum L., Xylosma longifolia Clos.

The major agreement to determine the most important plants in each ailment category was analyzed using fidelity level. Of the overall total 144 medicinal plants documented, 23 species were selected for all the categories which were used in the treatment of single or more than one ailment with multiple informants. The plants with less than three use reports were not considered for the analysis. The plant species with highest fidelity of $90 \%$ in single 
ailment were Justicia gendarrussa N.L. Burman, Lindernia ruellioides (Colsm.) Pennell and Dalbergia stipulacea Roxb.. Other plants with high fidelity level are Trichosanthes bracteata (Lam.) Voigt. with 80\%, Aloe vera Mill Gard. with $75 \%$, Justicia adhatoda L. with $72.72 \%$. Plants species with less than 3 use report were not considered for these analysis. Maximum FL indicated high preference and potential of healing among the informants for treating a particular ailment. The calculation also agrees with the Fic value of the present study. The frequently reported ailment Skeleton Muscular System Disorder (SMSD) had the highest Fic and Justicia gendarussa N.L. Burman used in the treatment of this particular ailment category also recorded the highest fidelity level of $90 \%$.

The plants with the highest fidelity level, use value and high informant consensus factor from the present findings indicates the possibility of high rate of occurrence of potential phytochemical compounds and therefore priority should be given to these plants to carry out phytochemical assays to study its efficacies.

\section{Conclusion:-}

Result of the present investigation advocates on the sustainable utilization of the plants so as to conserve the plant resources and also to blend the traditional knowledge with scientific findings. Such investigations need to be conserved as there are no written records and is passed on from their forefathers through orally. Further priority should be given to the plants with high use- value and fidelity level in developing novel drugs through detail clinical study.

\section{Acknowledgements:-}

The author is indebted to the local herbal practitioner and villagers for sharing their valuable ethnomedicinal knowledge, without their co-operation the work is not possible.

\section{References:-}

1. Abujam, S.K.S., Yumnam, R.S., Devi, O. Ch. and Chetia, D. (2012). Study on the Ethnomedicinal System of Manipur. International Journal of Pharmaceutical \& Biological Archives. 3(3): 587-591.

2. Angiosperm Phylogeny Group (APG) III. (2009). An Update of the Angiosperm Phylogeny Group classification for the orders and families of flowering plants. Botanical J. Linn. Soc. 161: 105-121.

3. Bendiksby, M., Thorbek, L., Scheen, A.C., Lindqvist, C. and Ryding ,O. (2011). An updated phylogeny and classification of Lamiaceae subfamily Lamioideae. Tax.; 60(2): 471-484.

4. Brummit, R.K. and Powell, C.E. (1992). Authors of plant names. A list of authors of scientific names of plants with recommended standard form of their names including abbreviations. Royal Botanic Garden, Kew. pp. 732.

5. Canales, M., Hernandez, T., Caballero, J., Romo de Vivar, A., Avila, G., Duran, A. and Lira, R. (2005). Informant consensus factor and antibacterial activity of the medicinal plants used by the people of San Rafael Coxcatlan, Puebla, Mexico. Journal of Ethnopharmavology. 97: 429-439.

6. Cook, F.E. (1995). Economic botany data collection standard. Royal Botanic Gardens (Kew).

7. Cox, A.P. and Ballick, J.M. (1994). The ethnobotanical approach to drug discovery. Scientific American. 82-87.

8. Cox, A.P. and Ballick, J.M. (1996). Ethnobotanical research and traditional healthcare in developing countries. Plant,People and Culture, New Work: Freeman, W.H. and Co.

9. Deb, D.B. (1961). Dicotyledonous plants of Manipur Territory. Bulletin Botanical Survey of India. p. 253-350.

10. Dutta, B.K. and Dutta, P.K. (2005). Potential of ethnobotanical studies in Northeast India: An overview. Indian Journal of Traditional Knowledge. 4: 7-14.

11. Friedman, J., Yaniv, Z., Dafni, A. and Palewitch, D. (1986). A preliminary classification of the healing potential of medicinal plants, based on a rational analysis of an ethnopharmacological field survey among Bedouins in the Nagev Desert, Israel. Journal of Ethnopharmacology. 16: 275-287.

12. Hamil, F.A., Apio, S., Mubiru, N.K., Mosango, M., Bukenya-Ziraba, R., Maganyi, O.W. \& Soejarto, D.D. (2000). Traditional herbal drugs of southern Uganda, I. Journal of Ethnopharmacology, 70: 281-300.

13. Heinrich, M., Ankli, A., Frei, B., Weimann, C. and Sticher, O. (1998). Medicinal plants in Mexico: healers' consensus and cultural importance. Social Science and Medicine. 47: 91-112.

14. Hooker, J.D. (1875). The Flora of British India, Vol I. Ranunculaceae to Sapindaceae. England: L. Reeve \& Co. Ltd.

15. Hooker, J.D. (1882). The Flora of British India, Vol III. Capeifoliaceae to Apocynaceae. England: L. Reeve \& Co. Ltd.

16. Li, X. and Hedge, I.C. (1994).In Wu ZY and Raven PH (eds.) Flora of China. Missouri Botanical Garden. 
17. Phillips, O. and Gentry, A.H. (1993). The useful plants of Tambopata, Peru: I, statistical hypotheses tests with a new quantitative technique. Economic Botany. 47: 15-32.

18. Phillips, O., Gentry, A.H., Reynel, C., Wilkin, P. and Galvez-Drand, B.C. (1994). Quantitative Ethnobotany and Amazonian conservation. Conservation Biology. 8: 225-248.

19. Pieroni, A. (2000). Medicinal plants and food medicines in the folk traditions of the upper Lucca Province, Italy, Journal of Ethnopharmacology, 70: 235-273.

20. Ragupathy, S., Steven, N.G., Maruthakkutti, M., Velusamy, B. and Ul-Huda, M.M. (2008). Consensus of the 'Malasars' traditional aboriginal knowledge of medicinal plants in the Velliangiri holly hills, India. Journal of Ethnobiology and Ethnomedicine. 4:8.

21. Reyes-Garcia, V., Huanca, T., Vadez, V., Leonard, W. and Wilkie, D. (2006). Cultural, practical and economic value of wild plants: a quantitative study in the Bolivian Amazon. Economic Botany. 60: 62-74.

22. Rokaya, M.B., Munzbergova, Z. and Timsina, B. (2010). Ethnobotanical study of medicinal plants from the Humla district of western Nepal. Journal of Ethnopharmacology. 130: 485-504.

23. Singh, N.P., Chauhan, A.S. and Mondal, M.S. (2000).Flora of Manipur, Vol 1. Calcutta: Botanical Survey of India.

24. Sur, P.R. and Halder, A.C. (2002). Observation on the Ethnobotany of Purulia District, West Bengal, India. In (eds.) Trivedi, P.C. Ethnobotany. Aavishkar Publishers, Distributors, Jaipur 302003 (Rajasthan) India. pp. 146168.

25. Trotter, R.T. and Logan, M.H. (1986). Informants consensus: a new approach for identifying potentially effective medicinal plants. In: Etkin, N.L. (Ed.), Plants in Indigenous Medicine and Diet. Redgrave Publishing Company, Bedford Hill, NY, pp. 91-112. 